\title{
EXPERIMENTAL ANALYSIS OF THE EFFECTS OF PARTICULATE FOULING ON HEAT EXCHANGER HEAT TRANSFER AND AIR SIDE PRESSURE DROP FOR A HYBRID DRY COOLER
}

\author{
I.H. Bell ${ }^{1}$, E.A. Groll ${ }^{2}$ and H. König ${ }^{3}$ and T. Odrich ${ }^{3}$ \\ ${ }^{1}$ Purdue University, 140 S. Martin Jischke Drive, West Lafayette, IN 47906 USA and ibell@purdue.edu \\ ${ }^{2}$ Purdue University, 140 S. Martin Jischke Drive, West Lafayette, IN 47906 USA \\ ${ }^{3}$ Jäggi/Güntner, Industriestrasse 23, CH-4632 Trimbach Switzerland
}

\begin{abstract}
It is well known that significant fouling by particulate matter can have a deleterious effect on the performance of enhanced surface heat exchangers, and the same is true for hybrid heat exchangers. Hybrid heat exchangers are heat exchangers which are typically run in dry mode to reject heat. When the ambient conditions require more heat rejection than can be provided by sensible heat transfer a water pump is turned on and water flows over the fins and the evaporation of water provides a further cooling effect. Fouling in dry mode operation is physically similar to that of air-cooled heat exchangers, but in evaporative mode the flow of the water over the coil eliminates the impact of fouling.

A hybrid dry cooler heat exchanger of $60 \mathrm{~cm} \times 60 \mathrm{~cm}$ frontal area has been installed in a well-instrumented wind tunnel to measure the heat exchanger's performance. Hot water flows through the coil to provide the load, and air flows over the coil to provide cooling. During evaporative mode operation another stream of water flows over the outside face of the coil, adhering mainly to the louvered fins. The louvered fins are specially designed for optimized water flow during wetting mode. The fins are made of aluminum, the tubes are copper, and protection against corrosion is realized by a special E-coating (König, 2006). This coil has been tested clean and fouled with ASHRAE standard dust, for both dry and wet operation. Results are presented for the air side pressure drop and overall heat transfer conductance of the coil under all conditions for which 50\% increases in air-side pressure drop are found under heavy fouling. The influence of fouling on heat transfer is small. Also, using the wetting water to wash the fouling off the coil is investigated, and found to be of some utility.
\end{abstract}

\section{INTRODUCTION}

The field of air-side fouling of HVAC heat exchangers has been previously studied, though nearly all is highly empirical and experimental in nature.

Ahn et al. (2003) investigated particulate fouling of tube-fin heat exchangers, for which they found that the pressure drop increased up to $45 \%$ over the duration of the tests. In addition, the heat transfer decreased by up to $14 \%$ with fouling, though it is not clear at what rate dust was injected into the air stream, nor the total amount of dust on the heat exchanger.

Lankinen et al. (2003) investigated the impact of airside fouling on the compact heat exchangers using dust similar to that under investigation here. They found increases in air-side pressure drop up to $200 \%$ and decreases in the overall heat transfer coefficient of $8 \%$ to $18 \%$.

Yang et al. (2007) carried out experiments on the fouling performance evaporators. They found that the pressure drop increased up to $30 \%$ with $300 \mathrm{~g}$ of dust, and the air-side heat transfer coefficient was sometimes improved, and sometimes decreased with fouling.

Siegel (2007) conducted research on the mechanics of particulate fouling as well as experimental measurements of system performance with fouling. It was found that the impact of fouling is a decrease in the air flow-rate of 5-6\% and a decrease in system efficiency of $2-4 \%$.

\section{EXPERIMENTAL PROCEDURE}

In order to fully characterize the effects of fouling on heat transfer and pressure drop in the heat exchanger, experimental measurements of the overall heat transfer conductance and air side pressure drop were carried out.

\section{Hybrid Dry Cooler Heat Exchanger}

The hybrid dry cooler shown in Figure 1 is a typical hybrid dry cooler in wetted mode. The investigated heat exchanger coil under study is a wetted hybrid coil with 9 tubes, each of which makes 8 passes through the fin sheet. The tubes layers are offset. The tube sheet is louvered in order to decrease the air-fin thermal resistance.

In dry mode, conditioned air flows over the finned tubes and warm water flows through the tubes. Heat transfer between hot water and cooler air causes the air temperature to increase and the water temperature to decrease. In dry mode, the pump which provides water to the wetting loop is left off.

When the coil is run in evaporative mode, wetting water is fed into the tray at the top of the heat exchanger. As the water overflows the tray, it drips down on the front face of 
the coil. The water that is not evaporated is then collected in the return tray at the bottom of the heat exchanger and, using a pump, pumped back into the feeding tray of the heat exchanger.

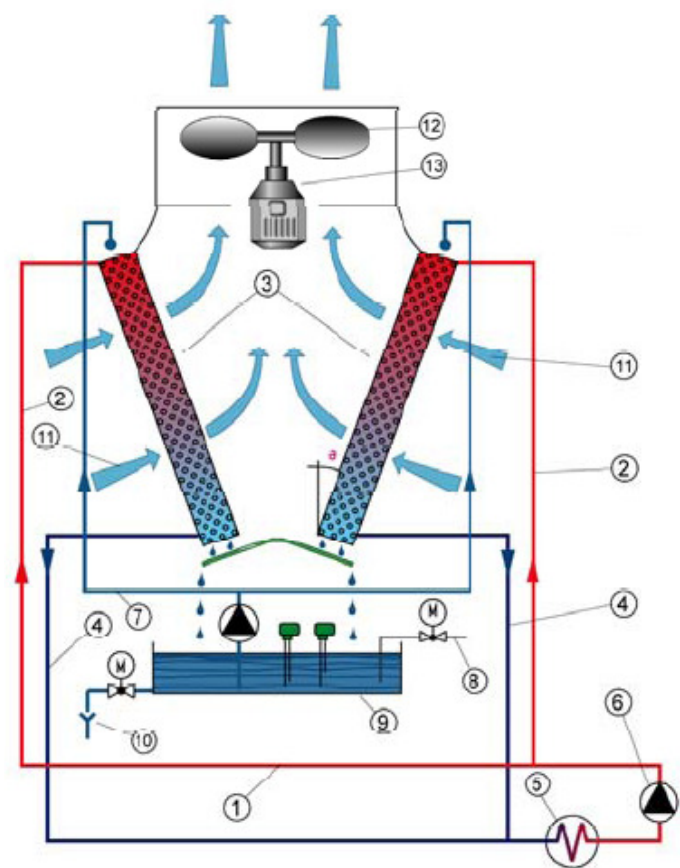

Figure 1 Schematic of hybrid dry cooler running in evaporative mode

\section{Particulate Fouling}

The particulate fouling employed in this study is ASHRAE standard 52.1 \#1 dust typically employed for testing of air filters and provided by Powder Technology Inc.. The composition of this dust is standardized, and the typical composition is shown in Table 1 .

Arizona test dust is primarily composed of $\mathrm{SiO}_{2}$ and $\mathrm{Al}_{2} \mathrm{O}_{3}$, all other components being each less than $5 \%$ by mass. The particle size distribution of Arizona test dust is shown in Figure 2.

Table 1 ASHRAE 52.1 \#1 Dust

\begin{tabular}{|cc|}
\hline Component & Percent Composition \\
\hline Arizona Test Dust & $72 \%$ \\
Carbon Black Powder & $23 \%$ \\
Milled Cotton Linters & $5 \%$ \\
\hline
\end{tabular}

\section{Testing Facility}

The testing facility used to carry out the measurements of the hybrid heat exchanger is shown in Figure 3.

Ambient air is drawn into the blower and the blower accelerates the air to the testing speed. Upon exiting the blower, the air passes over steam humidifying nozzles for which the steam flow rate is controlled by steam supply valves. The air then passes through a flow straightening section which aligns the bulk humidified flow, after which an array of pitot tubes are used to measure the air mean velocity.

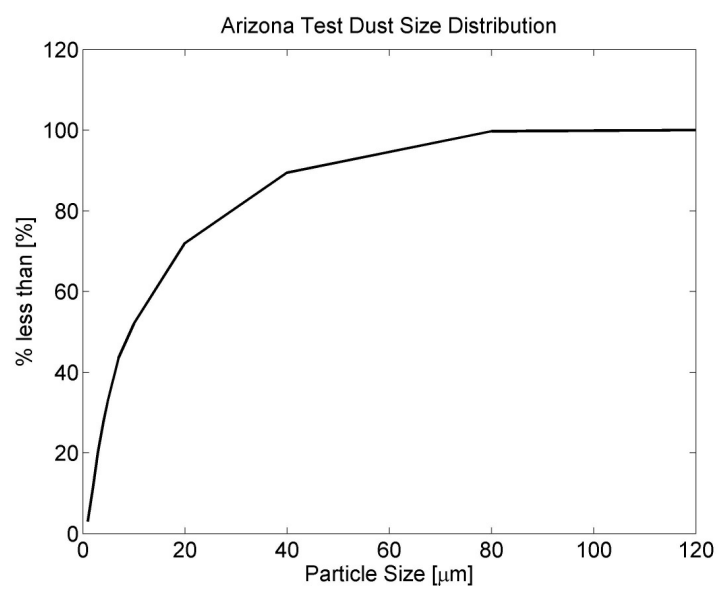

Figure 2 Arizona test dust particle size distribution

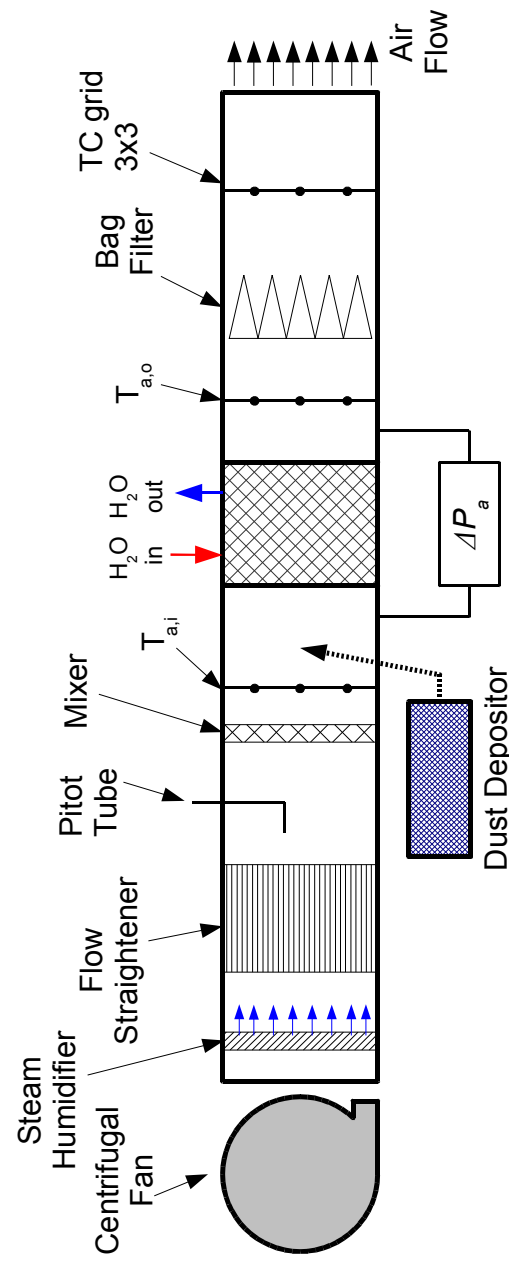

Figure 3 System Schematic

The air next flows through a mixing section to supply the coil with a uniform flow field. The next section of the duct is the dust injection section, in which dust can be injected into the air stream to foul the coil. The air then passes 
through the coil where it picks up heat, is filtered to remove any dust blown off the coil, and is exhausted to the ambient.

The water loop is comprised of a hot water heater of 9 $\mathrm{kW}$ rated power, a water pump to supply water to the heat exchanger, and several air purges distributed throughout the water loop in order to remove air introduced into the heat exchanger coils during charging.

\section{Testing Procedure}

Dry mode. The system schematic is seen in Figure 3. In order to characterize the performance of the heat exchanger the following nominal state points seen in Table 2 were achieved for clean, fouled and washed operation.

Table 2 Operating Points

\begin{tabular}{|cc|}
\hline Parameter & Value $(\mathrm{s})$ \\
\hline$T_{a, i}$ & $25.0 \pm 2.0^{\circ} \mathrm{C}$ \\
$T_{\mathrm{w}, \mathrm{i}}$ & $45.0 \pm 1.0^{\circ} \mathrm{C}$ \\
$\varphi_{i}$ & $40 \pm 10 \%$ \\
$\dot{m}_{w}$ & $0.172 \pm 0.010 \mathrm{~kg} / \mathrm{s}$ \\
$u_{\mathrm{a}}$ & $1.0,1.5,2.0,2.5,3.0 \pm 0.1 \mathrm{~m} / \mathrm{s}$ \\
\hline
\end{tabular}

The measured data values were acquired every 3 seconds. Acquisition was stopped when steady-state operation was achieved. After the clean data points were obtained, the fouling of the heat exchanger was carried out.

To foul the coil, a commercial dust injection machine was used which has a fixed aspirating head. Pre-massed amounts of dust were placed on the tray which was slowly fed towards the aspirating head. The dust aspirated by the dust injection machine enters the duct airstream, and subsequently is either deposited on the coil or captured by the filter which is downstream of the heat exchanger coil. The amount of dust captured by the air filter is calculated by measuring the mass of the filter before and after the fouling process. The remainder of the dust injected is assumed to be deposited on the coil.

Fouling was carried out at a nominal air velocity of 2.0 $\mathrm{m} / \mathrm{s}$ in two $300 \mathrm{~g}$ increments. Throughout the fouling process the air-side pressure drop increases due to the dust which deposits in the air filter and on the heat exchanger, and thus the blower power must be continually increased to maintain the air velocity of $2.0 \mathrm{~m} / \mathrm{s}$ during the fouling process.

After injecting $300 \mathrm{~g}$ of dust into the airstream the same state points shown in Table 2 were achieved and the steadystate performance was measured. The second $300 \mathrm{~g}$ of dust were subsequently injected, and the performance was again measured.

After the clean and fouled performances of the coil were measured, the cleaning procedure was carried out. To begin with, high pressure air was used to blow off all loose dust on the front and back faces of the heat exchanger. A second stage of washing was carried out by spraying distilled water on the front and back surfaces of the coil with a low-pressure hand-operated water sprayer. Macro photos of the coil fouled with $600 \mathrm{~g}$ of dust and washed are shown in Figure 4 and Figure 5 respectively.

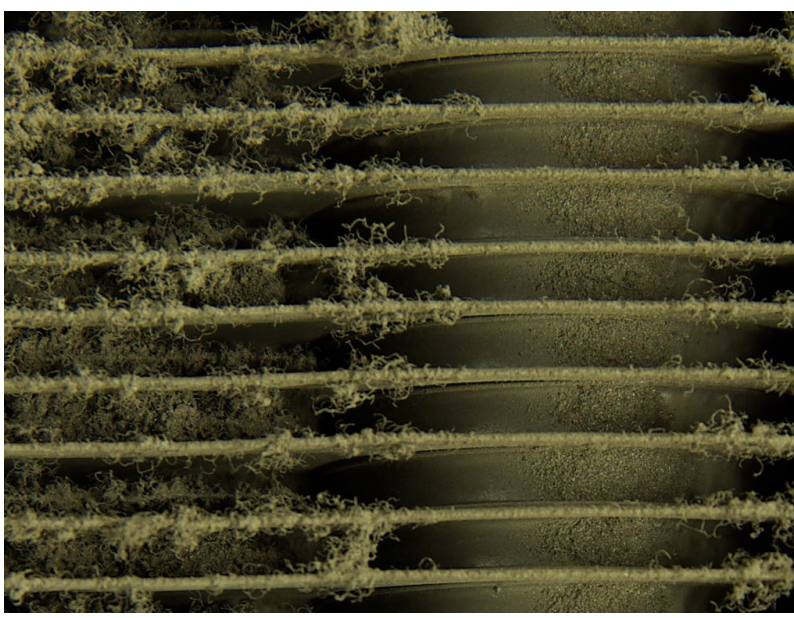

Figure 4 Front face of coil fouled with $600 \mathrm{~g}$ of injected dust

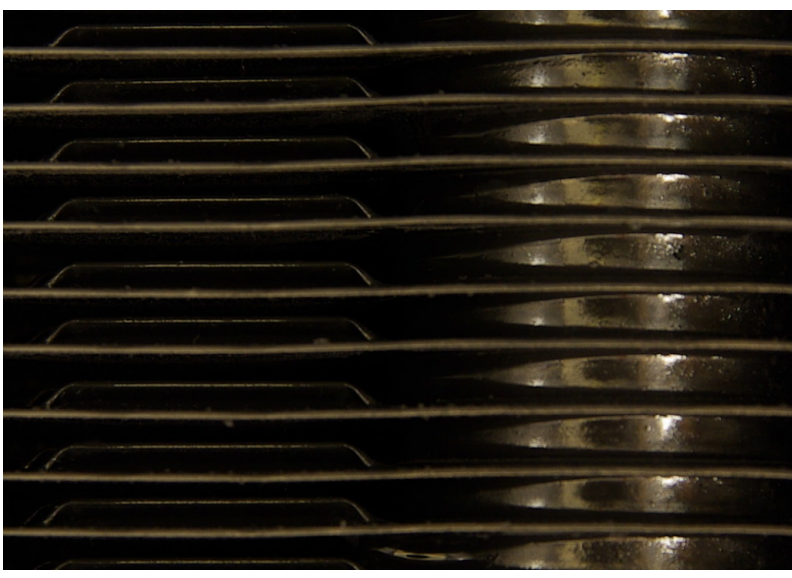

Figure 5 Front face of coil cleaned

Wetted Mode. In the wetted (evaporative) mode, the range of air velocities under interest is more restricted than the dry mode. The air velocity is limited at the low end by the ability to measure the air velocity, and the high end by entrainment. By entrainment this means that the velocity is high enough to strip the water off the coil and carry it downstream in droplet form. Thus the air velocities tested were $0.9 \mathrm{~m} / \mathrm{s}$ and $1.2 \mathrm{~m} / \mathrm{s}$. The volume flow rate of the wetting liquid was fixed at $720 \mathrm{~L} / \mathrm{h}$.

The cooling capacity of the coil at wetted condition equals approximately $60 \mathrm{~kW}$. Due to limitations of the heating power of the liquid loop, the water power was fixed at its maximum value of $9.0 \mathrm{~kW}$ for all of the wetted mode tests. The water flow rate was held the same as for the dry tests in order to have a uniform water-side thermal resistance for all the wetted and dry tests.

Fouling was carried out on the coil in wetted mode, but even after having injected $200 \mathrm{~g}$ of dust into the air stream, no air-side pressure drop increase was found. The decision was made to terminate the fouling process. 


\section{Data Reduction}

The air-side heat transfer is calculated based on measurement of the temperature and humidity at points upstream and downstream of the heat exchanger. Thus for "dry" operation the air-side heat transfer is given by

$q_{a}=\dot{m}_{a}\left(h_{a, o}-h_{a, i}\right)$

where the humid air enthalpies $h_{a, i}$ and $h_{a, o}$ are calculated based on the drybulb temperatures $T_{a, i}$ and $T_{a, o}$ and relative humidities $\varphi_{\mathrm{i}}$ and $\varphi_{\mathrm{o}}$ respectively. The air velocity $u_{a}$ is measured with a pitot-tube array by means of a differential pressure transducer. The air velocity is therefore given by

$u_{a}=\sqrt{\frac{2 \Delta p_{p}}{\rho_{a}}}$

where the density $\rho_{a}$ is based on the air inlet temperature and ambient pressure and $\Delta p_{p}$ is the pressure drop measured by the pitot-tube array. Both $T_{a, i}$ and $T_{a, o}$ are defined as the average air temperature measured over $3 \times 3$ grids of K-type thermocouples. The air mass flow rate is given by

$\dot{m}_{a}=\rho_{a} u_{a} A_{d}$

where the duct cross-sectional area $A_{d}$ is $0.37 \mathrm{~m}^{2}$. When the heat exchanger is run in evaporative mode, the amount of water evaporated is given by

$\dot{m}_{e}=\dot{m}_{a}\left(\omega_{o}-\omega_{i}\right)$

where the humidity ratios $\omega_{0}$ and $\omega_{i}$ are measured downstream and upstream of the heat exchanger respectively. Finally the air-side heat transfer (wetting water and air) is given by

$q_{a}=\dot{m}_{a}\left(h_{a, o}-h_{a, i}\right)-\dot{m}_{e} h_{e}+\dot{m}_{w w} c_{p, w w} \Delta T_{w w}$

where the evaporating water enthalpy $h_{e}$ is that for the water at the wetting water inlet temperature and ambient pressure. The sensible heat component is the right-most term in Eq. (5).

For the water loop, the heat transfer is given by

$q_{w}=\dot{m}_{w} c_{p, w}\left(T_{w, i}-T_{w, o}\right)$

where the mass flow $\dot{m}$ is measured with a Coriolis mass flow meter, and the inlet and outlet temperatures are measured with T-type thermocouples. The value of $c_{p, w}$ is based on the algebraic mean water temperature.

The log-mean temperature difference for the heat exchanger is given by
$\Delta T_{l m}=\frac{\left(T_{w, i}-T_{a, o}\right)-\left(T_{w, o}-T_{a, i}\right)}{\ln \frac{T_{w, i}-T_{a, o}}{T_{w, o}-T_{a, i}}}$

and thus the value of the effective heat transfer conductance $U A$ is given by

$U A=\frac{q}{\Delta T_{l m}}$

where the value of $q_{w}$ is used for the total heat transfer $q$ due to its lower uncertainty.

For the air-side pressure drop, the pressure drop is directly measured by means of a differential pressure transducer. In order to characterize the pressure drop for different conditions, a modified dimensional friction coefficient can be defined by

$\chi_{f}=\frac{\Delta p_{a}}{u_{a}^{2}}$

This friction factor is obtained for each operating condition by fitting experimental air-side pressure drop and air velocity data.

\section{Uncertainty Propagation}

The uncertainties of the measurement devices used in this study are given in Table 3 .

Table 3 Measurement Uncertainties

\begin{tabular}{|cc|}
\hline Parameter & Uncertainty \\
\hline$\Delta P_{p}$ & $0.025 \mathrm{~Pa}$ \\
$\Delta P_{a}$ & $0.25 \mathrm{~Pa}$ \\
$\mathrm{~T}_{\mathrm{a}, \mathrm{i}}, \mathrm{T}_{\mathrm{a}, \mathrm{o}}, \mathrm{T}_{\mathrm{w}, \mathrm{i}}, \mathrm{T}_{\mathrm{w}, \mathrm{o}}$ & $0.3 \mathrm{~K}$ \\
$\dot{m}_{w}$ & $0.4 \%$ \\
$\varphi_{i}, \varphi_{o}$ & $1 \%$ \\
\hline
\end{tabular}

The values of the uncertainties of the calculated values are shown in Figure 6 as a function of the air velocity for the cleaned coil. These calculated uncertainty values are representative of the uncertainties for all of the dry tests. From these results we see that the overall measurement uncertainty of the UA and $\Delta P_{a}$ values are in fact quite reasonable, even though the uncertainty of the air velocity and air-side heat transfer are quite high.

One caveat applies to the UA measurement uncertainties. Throughout the battery of tests that were carried out on this heat exchanger, it was found that guaranteeing a liquid loop free of air bubbles was nearly impossible. As a result, it is possible that the amount of residual air trapped in the liquid loop is not exactly the same for each test. This has a significant impact on the measured values of the overall heat transfer conductance though it has no impact on the air-side pressure drop. Thus the total uncertainty in UA is greater than the value calculated from the measurement uncertainties. 


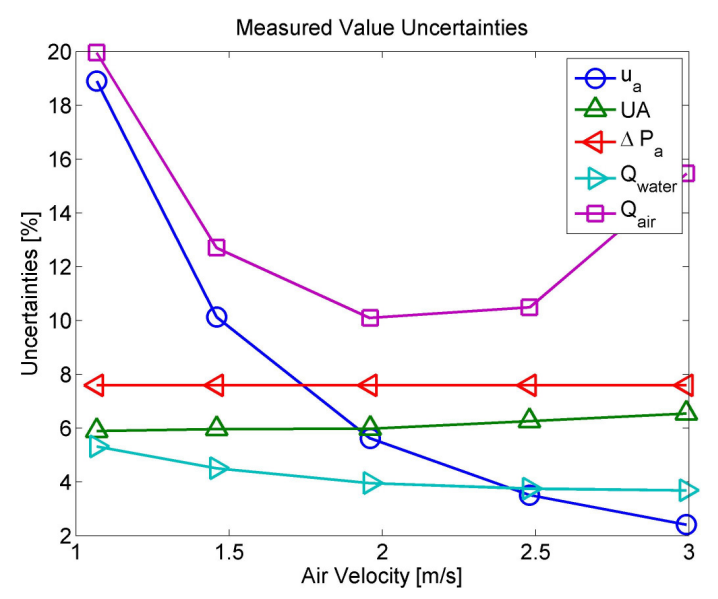

Figure 6 Measured value uncertainties for clean, dry coil

\section{RESULTS}

\section{Dry Operation}

For the dry coil, overall heat transfer conductance is seen in Figure 7. From this data it is clear that the impact of fouling on the coil heat transfer is small. The particulate fouling tends to build up on the front face of the fin sheets. Some of the fouling will undoubtedly be transported towards the interior of the heat exchanger and cause a dust film to cover the louver fin surfaces.

The impact of the dust covering the louvered fin surfaces does not necessarily have a negative effect. One of the components of the ASHRAE standard dust, carbon black, is a good thermal conductor. As a result, a thin coating of carbon black would tend to increase the effective surface area of the fins without causing a large increase in the fin-air thermal resistance.

As the fouling proceeds, more and more of the dust builds up on the finned surfaces, and ultimately its net effect is that it begins to constrict the heat transfer, resulting in a small decrease in the heat transfer. It should be noted that the uncertainties for the effective heat transfer conductances are quite large relative to the variation noted in the experimental data.

From the stand-point of pressure drop, fouling plays a much more significant and deleterious role during dry operation. For fixed air flow rate and inlet density, the fan pumping power is proportional to the pressure drop over the coil. Thus doubling the air-side pressure drop will also double the fan power required.

As seen in Figure 8, the impact of fouling on air-side pressure drop is quite significant. As the coil proceeds from the clean state to being fouled with $600 \mathrm{~g}$ of dust, the pressure drop increases significantly. After washing the coil, the pressure drop returns to slightly higher than the clean performance.

From the velocity-pressure drop data it is possible to determine the friction coefficient $\chi_{f}$ for the different coil states. Following this procedure, the friction coefficient was determined for the dry coil data. The normalized friction coefficients are presented in Figure 9. The friction coefficients were normalized by the friction coefficients for the clean case.

Thus it is clear just how deleterious the effects of airside particulate fouling are to air-side pressure drop. With $600 \mathrm{~g}$ of dust injected into the air stream, the friction coefficient increases by about $50 \%$. Thus if this level of fouling was allowed to be sustained in a practical application, the impact on fan power consumption would be significant.

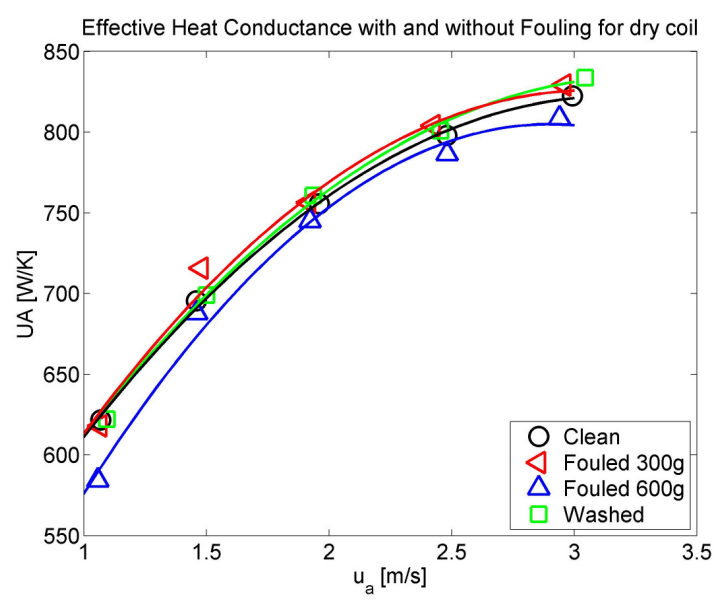

Figure 7: Heat conductance versus air velocity for dry operation

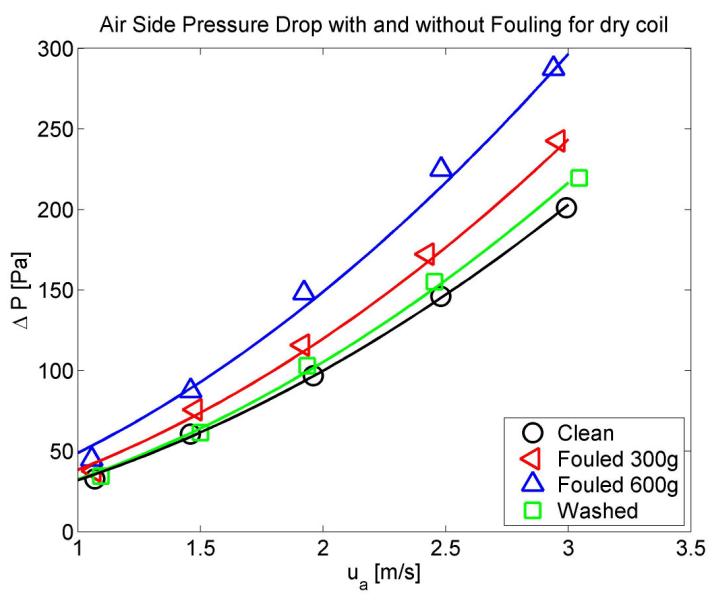

Figure 8: Air-side pressure drop for dry operation 


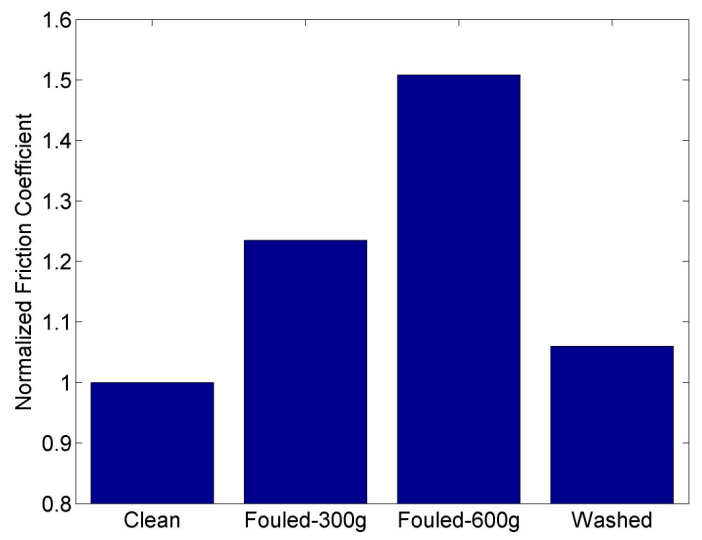

Figure 9: Normalized friction coefficients for dry operation

\section{Wetted Operation}

As with the operation of the coil in dry mode, the same parameters were measured for the coil with a wetting stream of liquid water.

In evaporative mode, the overall heat conductance is significantly higher because the wetting water can effectively use its latent heat to remove heat from the liquid loop. In addition the humid air entering the heat exchanger can absorb heat through sensible heat transfer as in the dry mode. In the evaporative mode, the mean humidity in the heat exchanger is higher and thus the mean mixture specific heat so the humid air can absorb more heat with less rise in temperature.

As a result, the overall heat transfer conductance measured in wetted mode is significantly higher than that of the dry mode for the same air velocity. As with the dry data, the overall heat transfer conductance increases with air velocity. This is due to improved heat and mass transfer between the air and the heat exchanger.

In wetted mode, the pressure drop is also higher, as seen in Figure 11. This is because the air tends to block off large portions of the front of the coil surface, which results in a larger relative velocity for the rest of the coil surface which then results in a larger pressure drop.

As discussed above, it was not possible to foul the coil in evaporative mode since no measureable increase in airside pressure drop was found after $200 \mathrm{~g}$ of particulate fouling had been applied to the heat exchanger.

\section{Wetted Washing}

One method proposed for cleaning the coil is to turn on the wetting loop after the coil has been fouled. To investigate this cleaning solution, the heat exchanger was fouled with $500 \mathrm{~g}$ of dust. Air-side pressure drop measurements were taken before and after fouling, as well as after washing the coil with clean wetting water without any cleaning agents or additives.

Using normal wetting water without cleaning agents to wash the coil was not a very effective means of cleaning the dust off the coil. In particular, the water left large pockets of the front face of the coil still fouled. In addition, the particulate fouling left on the coil would likely be more difficult to remove from the coil than without the wetting water washing.

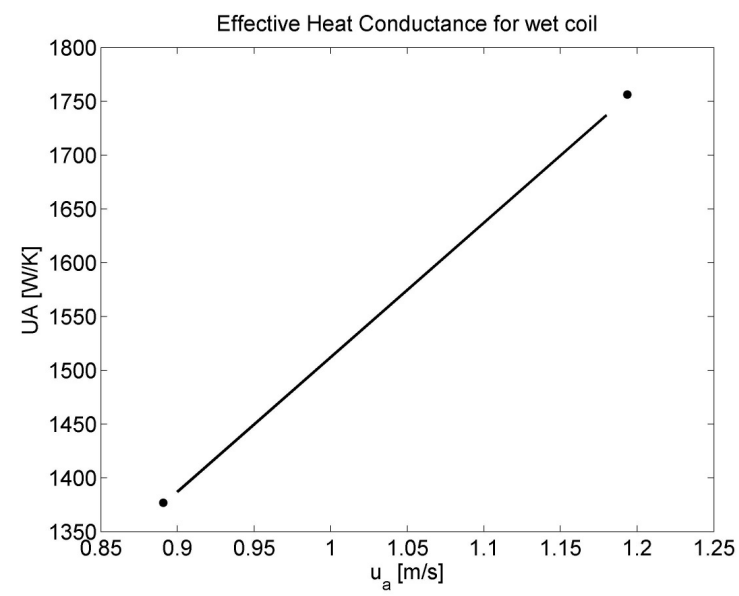

Figure 10 Heat conductance in evaporative mode

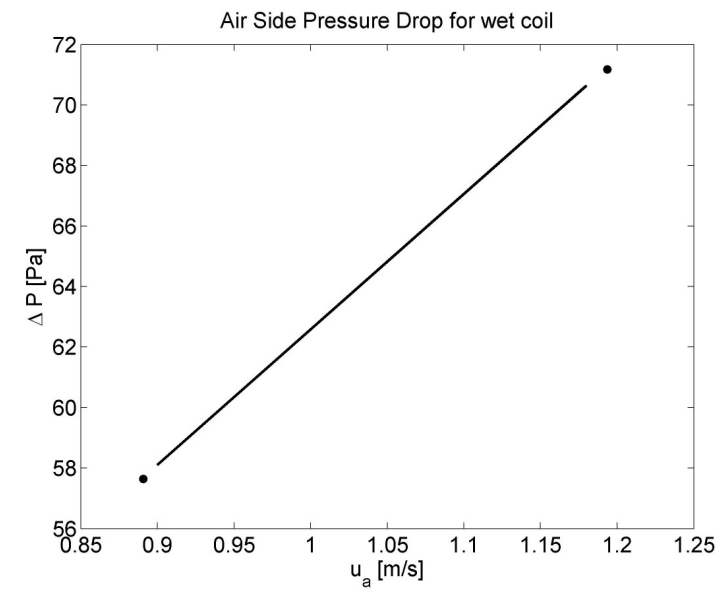

Figure 11 Air-side pressure drop in evaporative mode

The pressure drop data also shows that the effects of the wetting water washing are not very significant. Based on the velocity-pressure drop data, the frictional coefficient is calculated for the different heat exchanger statuses, the results of which are shown in Figure 12. The conclusion is that using normal wetting water without cleaning agents to rinse the particulate fouling off the coil is an ineffectual method for cleaning a fouled heat exchanger. This method of cleaning the coil will be investigated in the next part of the study.

The poor cleaning performance of the wetting water washing is largely due to the fact that the cleaning water needs additional additives and cleaning agents. The cleaning agents used in typical operation are selected according to the local water conditions, of which the most important parameters are the water hardness and chlorine concentration. 


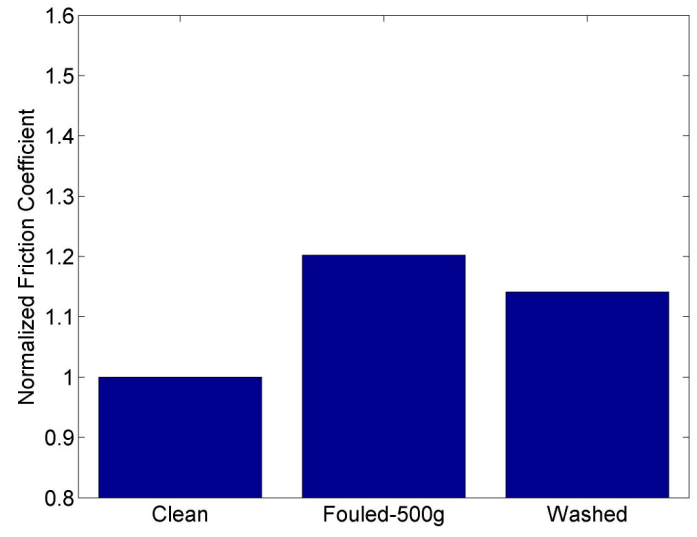

Figure 12 Normalized friction coefficient for wetting water cleaning by unconditioned distilled wetting water

\section{CONCLUSIONS}

From the study carried out above, the following conclusions are possible:

1. Air-side particulate fouling results in a significant increase in the air-side pressure drop of up to $50 \%$ with $600 \mathrm{~g}$ of fouling, but has little impact on the thermal performance

2. Measured frictional coefficient is proportional to amount of fouling applied over the range of fouling investigated.

3. In evaporative mode, fouling has no measured impact on air-side pressure drop or heat transfer performance. This is one of the primary benefits of hybrid dry cooling units in locations with a high content of dust during typical operation.

4. Using only distilled wetting water is not an effective means of washing the particulate fouling off of the heat exchanger. Effective cleaning requires conditioned water with the appropriate additives.

\section{NOMENCLATURE}

$\begin{array}{lll}\mathrm{A} & \text { Area } & \mathrm{m}^{2} \\ \chi_{\mathrm{f}} & \text { Frictional coefficient } & \mathrm{Pa}-\mathrm{s}^{2} / \mathrm{m}^{2} \\ \mathrm{c}_{\mathrm{p}} & \text { Specific heat } & \mathrm{kJ} / \mathrm{kg} \mathrm{K} \\ \mathrm{h} & \text { Enthalpy } & \mathrm{kJ} / \mathrm{kg} \\ \dot{m} & \text { Mass flow rate } & \mathrm{kg} / \mathrm{s} \\ \Delta \mathrm{p} & \text { Pressure drop } & \mathrm{Pa} \\ \mathrm{q} & \text { Heat transfer rate } & \mathrm{kW} \\ \mathrm{T} & \text { Temperature } & { }^{\circ} \mathrm{C} \\ \mathrm{u} & \text { Velocity } & \mathrm{m} / \mathrm{s} \\ \mathrm{UA} & \text { Overall heat transfer conductance } & \mathrm{kW} / \mathrm{K} \\ & & \\ \varphi & \text { Relative Humidity } & \% \\ \rho & \text { Density } & \mathrm{kg} / \mathrm{m}^{3} \\ \omega & \text { Humidity Ratio } & \mathrm{kg} / \mathrm{kg}\end{array}$

\begin{tabular}{ll}
\multicolumn{2}{l}{ Subscripts } \\
a & Humid Air \\
$\mathrm{d}$ & Duct \\
$\mathrm{e}$ & Evaporated \\
$\mathrm{i}$ & Inlet \\
$\mathrm{lm}$ & Log-mean \\
$\mathrm{o}$ & Outlet \\
$\mathrm{p}$ & Pitot-tube \\
$\mathrm{w}$ & Water \\
ww & Wetting water
\end{tabular}

\section{REFERENCES}

H. König, 2006, Simulation and Operation of Hybrid Dry Coolers at Part Load conditions, $7^{\text {th }}$ VDMA Conf. Cooling Towers, 9.11.2006, Frankfurt, Germany

Y.-C. Ahn, J.-M. Cho et al., 2003, An Experimental Study of the Air-side Particulate Fouling in Fin-and-Tube Heat Exchangers of Air Conditioners, Korean J. Chem. Eng., Vol. 20, pp. 873-877.

R. Lankinen, J. Suihkonen, P. Sarkomaa, 2003, The effect of air side fouling on thermal-hydraulic characteristics of a compact heat exchanger, Int. J. Energy Res., Vol. 27, pp. 349-361.

J.A. Siegel, 2002, Particulate Fouling of HVAC Heat Exchangers, Ph.D Thesis

L. Yang, J.E. Braun, E.A. Groll, 2007, The impact of fouling on the performance of filter-evaporator combinations, Int. J. Refrig., Vol. 30, pp. 498-498. 